Instability, Chaos and Predictability

in Celestial Mechanics and

Stellar Dynamics
Copyright 1992 by Nova Science Publishers, Inc. All rights of reproduction in any form reserved. ISBN 1-56072-054-9

\title{
Algorithms for CONTROLling ChaOs: APPLICATION TO BVP OSCILlATOR
}

\author{
S. Rajeskar \\ Senior Research Fellow, Dept. of Physics, Bharathidasan University, \\ Tiruchirapallin - 620024, Tamilnadu
}

\begin{abstract}
We discuss how chaotic dynamics can be converted into regular motion in Bonhoffer-van der Pol osillator. Using a control signal proportional to the actual and desired outputs we study the control of $f$ ixed points and 1 imit cycles by making time-dependent perturbations of amplitude of exter nal force. he show the round off induced periodicity in the digital computer simulations of orbits on chaotic attractor. We illustrate the stabilization of unstable periodic or bits by adding periodic pulses of mall magnitude.
\end{abstract}

